\title{
Prognostic value of cytokines and chemokines in addition to the GRACE Score in non-ST-elevation acute coronary syndromes
}

\author{
Luis C.L. Correia b,c,*, Bruno B. Andrade ${ }^{a}$, Valéria M. Borges ${ }^{a}$, Jorge Clarêncio ${ }^{a}$, Ana P. Bittencourt ${ }^{\text {b }}$, \\ Rafael Freitas $^{\mathrm{b}}$, Alexandre C. Souza ${ }^{\mathrm{b}}$, Maria C. Almeida ${ }^{\mathrm{b}}$, Jamile Leal ${ }^{\mathrm{b}}$, J. Péricles Esteves ${ }^{\mathrm{c}}$, \\ Manoel Barral-Netto ${ }^{\mathrm{a}, \mathrm{d}}$ \\ a Gonçalo Moniz Research Center, Oswaldo Cruz Foundation (FIOCRUZ), Bahia, Brazil \\ b Cardiology Division, Portuguese Hospital, Salvador/BA, Brazil \\ ${ }^{c}$ Medical School of Bahia, Salvador/BA, Brazil \\ d Institute of Investigation in Immunology (iii), Science and Technology National Institute (INCT), Bahia, Brazil
}

\section{A R T I C L E I N F O}

\section{Article history:}

Received 21 October 2009

Received in revised form 21 December 2009

Accepted 7 January 2010

Available online 18 January 2010

\section{Keywords:}

Cytokines

Chemokines

C-reactive protein

Acute coronary syndromes

\begin{abstract}
A B S T R A C T
Background: Increased cytokine and chemokine levels are associated with cardiovascular events in patients with non-ST-elevation acute coronary syndromes (ACS), but the incremental prognostic value of these inflammatory markers is not known. We determined if cytokine and chemokine assessment adds prognostic information to the GRACE Score in patients with ACS.

Methods: Five cytokines (interleukin (IL)-1 $\beta$, IL-6, IL-10, IL-12p70, and tumor necrosis factor (TNF)- $\alpha$ soluble receptor I), five chemokines (IL-8, CCL5, CXCL9, CCL2, and CXCL10) and C-reactive protein (CRP) were measured at admission of 87 patients admitted with ACS.

Results: During hospitalization, the incidence of cardiovascular events was 13\% (7 deaths, 1 nonfatal acute myocardial infarction, and 3 refractory unstable angina). Individuals who developed events had significantly greater levels of CRP, IL-1 $\beta$, IL-12, TNF- $\alpha$, IL-8, CXCL9 and CCL2, compared with those free of events. Thus, these markers were used to build an Inflammatory Score, by the input of one point for each of these variables above the 75th percentile. After adjustment for the GRACE Score, the Inflammatory Score independently predicted events $(\mathrm{OR}=1.80 ; 95 \% \mathrm{CI}=1.12-1.88)$. Incorporation of the Inflammatory Score into the GRACE Score promoted a C-statistics improvement from 0.77 ( $95 \% \mathrm{CI}=0.58-0.96)$ to 0.85 (95\% $\mathrm{CI}=0.71-1.0)$. Net reclassification improvement obtained with GRACE-Inflammatory Score was $13 \%$ $(P=0.007)$, indicating a significant reclassification. When only CRP was incorporated into GRACE, the increase on C-statistics was not relevant (from 0.77 to 0.80 ).

Conclusion: Cytokines and chemokines measured at admission add prognostic information to the GRACE Score in patients admitted with ACS.
\end{abstract}

(c) 2010 Elsevier B.V. All rights reserved.

\section{Introduction}

Individuals admitted with non-ST elevation acute coronary syndromes (ACS) have a wide range of risk regarding recurrent events during hospitalization. Since the benefits of investigational and therapeutic strategies vary with baseline risk, it is important to stratify patients according to the probability of adverse outcome. The most reliable method to assess individual risk at admission is the utilization of risk scores [1], which takes into consideration the severity of clinical presentation, age and co-morbidities. Among validated scores, the GRACE Score has been established as the most accurate $[2,3]$.

\footnotetext{
* Corresponding author. Av. Princesa Leopoldina, 19/402, 40.150-080, Salvador, BA Brazil. Tel.: +55 713263 3584; fax: + 557132633584 .

E-mail address: lccorreia@terra.com.br (L.C.L. Correia).
}

Inflammation is recognized as an important mechanism of plaque destabilization and inflammatory markers are demonstrated to be predictors of recurrent events in ACS, independent of traditional risk markers [4,5]. However, to become an established tool in risk stratification, in addition to being an independent predictor, a biomarker needs to convincingly demonstrate its incremental utility beyond that of existing predictive models [6]. We previously demonstrated that C-reactive protein (CRP) adds predictive value to the TIMI-Risk Score in patients with non-ST elevation ACS [7]. However, the inflammatory process that underlines atherosclerosis is mediated by a multitude of cytokines and is unlikely to be totally reflected by CRP. Therefore, it is possible that the assessment of cytokines and chemokines improves risk prediction in ACS. Although several cytokines and chemokines have been associated with recurrent events in ACS, the incremental prognostic value of measuring a panel of these markers in addition to risk scores has not been tested. 


\section{Methods}

\subsection{Study population}

Consecutive patients admitted to the coronary care unit of Portuguese Hospital due to unstable angina pectoris or non-ST elevation acute myocardial infarction between August 2007 and August 2008 were considered candidates for the study. Inclusion criteria were defined as onset of typical chest discomfort at rest in the prior $48 \mathrm{~h}$, in the absence of ST-segment elevation and the presence of at least one of the following objective criteria: (1) positive serum marker of myocardial necrosis, defined as troponin $\mathrm{T} \geq 0.01 \mu \mathrm{g} / \mathrm{l}$, which corresponds to the values above the 99th percentile of a healthy reference population [8]; (2) electrocardiographic ischemic changes consisting of transient ST-segment depression $(\geq 0.05 \mathrm{mV})$ or T wave inversion $(\geq 0.1 \mathrm{mV})$; (3) previous documentation of coronary artery disease, defined as a history of myocardial infarction or coronary obstruction $\geq 50 \%$ at angiography. Once fulfilling inclusion criteria, all patients that provided informed consent were included in the study. The study protocol conforms to the ethical guidelines of the 1975 Declaration of Helsinki as reflected in a priori approval by the Institution's human research ethic committee.

\subsection{Study protocol}

A blood sample to measure cytokines, chemokines and CRP was drawn immediately at hospital arrival, targeting the least possible time delay from symptoms onset. Plasma markers of myocardial necrosis were evaluated initially at hospital arrival and $6 \mathrm{~h}$ later. The index diagnosis was defined as non-ST elevation acute myocardial infarction when troponin $\mathrm{T} \geq 0.03 \mu \mathrm{g} / \mathrm{l}$, which is the $10 \% \mathrm{CV}$ cut-off [8]. The remaining subjects were labeled as unstable angina.

As the primary end-point, combined cardiovascular events during hospitalization were defined as the composite of death, nonfatal acute myocardial infarction or refractory unstable angina. Myocardial infarction as an outcome endpoint was defined as either a new Q-wave or troponin elevation during hospitalization despite normal values during the first $24 \mathrm{~h}$. For patients with infarction at admission, a new peak of mass CK-MB ( $>50 \%$ the previous value and above the normal value) was required for the diagnosis of reinfarction. Refractory angina during hospitalization was defined as recurrent chest pain at least twice, despite nitrates and anti-angina therapy ensuring controlled oxygen consumption.

\subsection{Clinical risk assessment}

In order to assess risk of recurrent events by clinical information, the GRACE Score was utilized. GRACE calculation was based on admission data and performed as previously validated [9]. Briefly, this score consists of 8 variables, 5 semi-quantitative (age, systolic blood pressure, heart rate, plasma creatinine, and Killip class) and 3 dichotomic variables (positive necrosis markers, ST-segment deviation and cardiac arrest at admission). Points are attributed according to the values of each variable and the sum of all variables corresponds to a previously defined risk level.

\subsection{Inflammatory markers}

Plasma inflammatory cytokines and chemokines were measured respectively by $\mathrm{BD}^{\mathrm{TM}}$ Cytometric Bead Array Human Inflammatory Kit (interleukin (IL)-1ß, IL-6, IL-10 and IL-12p70) [10] and $\mathrm{BD}^{\mathrm{TM}}$ Cytometric Bead Array Human Chemokine Kit (IL-8, CCL5/RANTES, CXCL9/MIG, CCL2/MCP-1, and CXCL10/IP-10) [10], according to the manufacturer's instructions (BD Biosciences, San Jose, CA). Cytometric Bead Array allows simultaneous protein analysis by flow cytometry. The concentrations of samples were calculated by extrapolating the mean fluorescence intensity (MFI) on the respective standard curves. Tumor necrosis factor (TNF)- $\alpha$ soluble receptor I was measured by ELISA (R\&D Systems, Inc. Minneapolis, MN) [11]. Plasma C-reactive protein was assessed by the commercially available high-sensitivity method of nephelometry (Dade-Behring, Newark, DE) [12].

\subsection{Data analysis}

In order to test the principal hypothesis, the following procedures were applied: (1) after log-transformation, inflammatory markers were compared between individuals who developed subsequent events and those free of events by Student's $t$ test. In case logtransformation did not provide a normal distribution, Wilcoxon's Rank-sum test was used; (2) the markers significantly associated with events were selected to constitute an Inflammatory Score. In each patient, the inflammatory variables were considered positive when the value was above the 75th percentile. For each positive variable, one point was added to the Score; (3) logistic regression analysis was used to evaluate the predictive value of the Inflammatory Score after adjusting for the GRACE Score and other potential confounding variables; (4) predicted probabilities of the model containing both GRACE and Inflammatory Scores were generated by logistic regression analysis. Discrimination ability of this model was assessed by the area under the curve of predicted probabilities (C-statistics); (5) the multivariate model was translated into a GRACE-Inflammatory Score after multiplying the Inflammatory Score of each patient by the ratio of Inflammatory Score's regression coefficient/GRACE Score's regression coefficient in logistic regression. By doing this, the weight attributed to inflammation into the GRACE Score was proportional to its weight in the multivariate model. Then, the point of best accuracy in the ROC curve was identified in each score and used to calculate sensitivity, specificity and predictive values. Sensitivities and specificities were compared between scores by McNemar's test; (6) finally, reclassification tables were built and net reclassification improvement [13] was utilized to evaluate whether the new score correctly reclassifies patients according to low or high risk. In addition, Integrated Discrimination Improvement was calculated [13]. The null hypothesis of no reclassification was tested under the error $\alpha$ of 0.05 .

Considering the non-normal distribution of inflammatory markers, medians and interquartile ranges (IQR) were used to describe cytokines, chemokines and CRP. Normally distributed numeric variables were expressed in mean \pm standard deviation and categorical variables in relative frequencies. In general, Pearson's $\chi^{2}$ test was used to compare categorical variables, Student's $t$ test to compare normally distributed variables, Wilcoxon's Rank-sum test to compare non-normally distributed variables between groups, and Spearman correlation to assess linear association between variables. Statistical significance was defined as a $P$ value $<0.05$. SPSS Statistical Software (ver 9.0, SPSS Inc., Chicago, IL) and MedCalc Statistical Software (ver 9.3.2.0, MedCalc Software, Mariakerke, Belgium) were used for data analysis.

\section{Results}

\subsection{Population characteristics}

Eight-seven patients were studied, aged $71 \pm 12$ y, 55\% females, $63 \%$ diagnosed as non-ST-elevation infarction and the remaining as unstable angina. Time elapsed between symptoms onset and blood draw for inflammatory markers had a median of $4.3 \mathrm{~h}$ (IQR 3-12 h). During a median hospitalization of 8 days (IQR 6-18 days), the incidence of cardiovascular events was 13\% (7 deaths, 1 nonfatal acute myocardial infarction or 3 patients with refractory unstable angina). Individuals with events presented a greater GRACE Score (median 182; IQR 149-227), in comparison with those free of events (median 
118; IQR 105-158; $P=0.004)$. The components of GRACE significantly associated with events were positive troponin $\mathrm{T}$, plasma creatinine and Killip class. In addition, males were more common in patients with events, as well as triple vessel or left main disease - Table 1 .

\subsection{Inflammatory markers and cardiovascular events}

Individuals who developed cardiovascular events during hospitalization had greater levels of CRP (median $17 \mathrm{mg} / \mathrm{l}$ vs. $5.3 \mathrm{mg} / \mathrm{l}$, $P=0.02), \mathrm{IL}-1 \beta$ ( $12 \mathrm{pg} / \mathrm{ml}$ vs. $7.3 \mathrm{pg} / \mathrm{ml}, P=0.05), \mathrm{IL}-12$ ( $43 \mathrm{pg} / \mathrm{ml}$ vs. $5.6 \mathrm{pg} / \mathrm{ml}, P=0.03)$, TNF- $\alpha$ soluble receptor I $(2.416 \mathrm{pg} / \mathrm{ml} v s$. $1.700 \mathrm{pg} / \mathrm{ml}, P=0.01), \mathrm{IL}-8$ ( $37 \mathrm{pg} / \mathrm{ml}$ vs. $10 \mathrm{pg} / \mathrm{ml}, P=0.003)$, CXCL9 $(1.396 \mathrm{pg} / \mathrm{ml}$ vs. $513 \mathrm{pg} / \mathrm{ml}, P=0.02)$ and CCL2 $(183 \mathrm{pg} / \mathrm{ml}$ vs. $145 \mathrm{pg} / \mathrm{ml}, P=0.02$ ), compared with those free of events. As opposed, the levels of IL-6, IL-10, CCL5 and CXCL10 were similar between the two groups - Table 2 .

Therefore CRP, IL-1, IL-12, TNF- $\alpha$ soluble receptor I, IL-8, CXCL9 and CCL2 were used to build the Inflammatory Score, by the input of one point for each of these variables above the 75th percentile of the sample distribution. This score had a significantly higher median of 4 (IQR 2-4) in patients with events, compared to a median of 1 (IQR 0-2) in individuals with no events $(P<0.001)-$ Fig. 1 .

\subsection{Adjustment for necrosis and systolic function}

The Inflammatory Score was correlated with peak troponin $\mathrm{T}$ $(r=0.40 ; P<0.001)$ and peak CK-MB $(r=0.28 ; P=0.01)$, suggesting that necrosis enhances inflammation. Thus, we used logistic regression to specifically adjust the predictive value of the Inflammatory Score for peak TnT and peak CK-MB, entered as continuous variables. In the first model, the Inflammatory Score remained a significant predictor $(\mathrm{OR}=1.9 ; 95 \% \mathrm{CI}=1.2-3.1 ; P=0.007)$, after adjustment for peak $\operatorname{TnT}(\mathrm{OR}=2.6 ; 95 \% \mathrm{CI}=1.2-5.6 ; P=0.02)$. Seemingly, the Inflammatory Score remained a significant predictor $(\mathrm{OR}=2.0 ; 95 \%$ $\mathrm{CI}=1.3-3.2 ; P=0.003)$, after adjustment for peak $\mathrm{CK}-\mathrm{MB}(\mathrm{OR}=1.01$; $95 \% \mathrm{CI}=0.99-1.03 ; P=0.27)$. Therefore, we conclude that the prognostic value of the Inflammatory Score takes place beyond the necrosis phenomenon.

Table 1

Comparison of clinical characteristics between patients who developed events and those free of events.

\begin{tabular}{|c|c|c|c|}
\hline & No events & Events & $P$ value \\
\hline Sample size & 76 & 11 & \\
\hline \multicolumn{4}{|l|}{ Clinical characteristics } \\
\hline Age $(y)$ & $71 \pm 12$ & $73 \pm 9$ & NS \\
\hline Males & $31(41 \%)$ & $8(73 \%)$ & NS \\
\hline ST-segment depression & $10(13 \%)$ & $2(18 \%)$ & NS \\
\hline Positive troponin $\mathrm{T}$ & $33(43 \%)$ & $9(82 \%)$ & 0.02 \\
\hline Troponin $\mathrm{T}(\mu \mathrm{g} / \mathrm{l})$ & $0(0-0.07)$ & $0.12(0.02-0.36)$ & 0.003 \\
\hline Plasma creatinine $(\mathrm{mg} / \mathrm{dl})$ & $1.0(0.85-1.2)$ & $1.6(1.2-2.3)$ & 0.001 \\
\hline Systolic blood pressure $(\mathrm{mmHg})$ & $157 \pm 37$ & $150 \pm 41$ & NS \\
\hline Heart rate (bpm) & $75 \pm 17$ & $90 \pm 25$ & NS \\
\hline Killip $>1$ & $16(21 \%)$ & $6(55 \%)$ & 0.02 \\
\hline LV ejection fraction $<45 \%$ & $8(11 \%)$ & $2(18 \%)$ & NS \\
\hline Triple vessel disease or LM & $18(35 \%)$ & $6(86 \%)$ & 0.02 \\
\hline GRACE Score & $118(105-158)$ & $182(149-227)$ & 0.004 \\
\hline \multicolumn{4}{|l|}{ Pharmacological and invasive therapy } \\
\hline Previous aspirin & $31(43 \%)$ & $5(46 \%)$ & NS \\
\hline Previous statin & $19(27 \%)$ & $4(40 \%)$ & NS \\
\hline Aspirin & 75 (99\%) & $10(91 \%)$ & NS \\
\hline Clopidogrel & $71(93 \%)$ & $9(82 \%)$ & NS \\
\hline IIb/IIIa receptor blockers & $4(5.3 \%)$ & $2(18 \%)$ & NS \\
\hline Enoxaparin & $68(90 \%)$ & $9(82 \%)$ & NS \\
\hline Statins & $70(93 \%)$ & $11(100 \%)$ & NS \\
\hline Coronary angioplasty & $16(21 \%)$ & $6(55 \%)$ & 0.02 \\
\hline Coronary bypass surgery & $3(4.0 \%)$ & $2(18 \%)$ & NS \\
\hline
\end{tabular}

Table 2

Comparison of inflammatory markers between patients who developed events and those free of events.

\begin{tabular}{|c|c|c|c|}
\hline Inflammatory marker & No events & Events & $P$ value \\
\hline Sample size & 76 & 11 & \\
\hline C-reactive protein (mg/l) & $5.3(1.5-11)$ & $17(3.2-91)$ & 0.02 \\
\hline \multicolumn{4}{|l|}{ Cytokines } \\
\hline IL-1及 (pg/ml) & $7.3(6.0-13)$ & $12(7.2-19)$ & 0.05 \\
\hline IL-6 (pg/ml) & $5.9(4.7-11)$ & $7.8(5.5-19)$ & NS \\
\hline IL-10 (pg/ml) & $6.9(5.5-7.8)$ & $8.6(4.5-13)$ & 0.21 \\
\hline IL-12 (pg/ml) & $5.6(4.4-31)$ & $43(5.2-54)$ & 0.025 \\
\hline TNF- $\alpha(p g / m l)$ & $1,700(1,252-2,609)$ & $2,416(1,216-5,547)$ & 0.01 \\
\hline \multicolumn{4}{|l|}{ Chemokines } \\
\hline IL-8 (pg/ml) & $10(6.2-28)$ & $37(30-46)$ & 0.003 \\
\hline CCL5 (pg/ml) & $43,635(42,893-43,902)$ & $43,906(43,013-44,044)$ & NS \\
\hline CXCL9 (pg/ml) & $513(294-1,162)$ & $1,396(506-28,060)$ & 0.02 \\
\hline CCL2 (pg/ml) & $145(123-165)$ & $183(122-191)$ & 0.02 \\
\hline CXCL10 $(\mathrm{pg} / \mathrm{ml})$ & $302(175-265)$ & $540(190-1.032)$ & NS \\
\hline
\end{tabular}

Values are expressed in medians and interquartile ranges.

Statistical comparison between the two groups was performed by Student's $t$ test after log-transformation or Mann-Whitney test if non-normal distribution persisted.

IL: interleukin; TNF- $\alpha$ : tumor necrosis factor soluble receptor I.

The Inflammatory Score was also correlated with Killip Class $(r=0.31 ; P=0.004)$, indicating that systolic dysfunction might influence the inflammatory process. Thus, we specifically adjust the predictive value of the Inflammatory Score for systolic dysfunction by logistic regression. In the first model, the Inflammatory Score remained a significant predictor $(\mathrm{OR}=1.9 ; 95 \% \mathrm{CI}=1.2-3.0$; $P=0.007)$, after adjustment for Killip Class $(P=0.21)$. In the second model, the Inflammatory Score remained a significant predictor ( $\mathrm{OR}=2.1 ; 95 \% \mathrm{CI}=1.3-3.3 ; P=0.001)$, after adjustment for systolic dysfunction $(\mathrm{FE}<45 \%)$ assessed by echocardiography $(P=0.68)$. Therefore, we can state that systolic dysfunction was not a confounding factor in the association between inflammation and cardiovascular events.

\subsection{Independent prognostic value of inflammation}

In a logistic regression analysis, the Inflammatory Score was adjusted for the GRACE Score and gender. Gender did not maintain statistical significance after adjustment for the other two covariates $(P=0.13)$ and was kept out of the final model. The Inflammatory Score independently predicted events ( $\mathrm{OR}=1.80 ; 95 \% \mathrm{CI}=1.12$ 1.88) after adjustment for GRACE, which was also significant in the final model $(\mathrm{OR}=1.02 ; 95 \% \mathrm{CI}=1.003-1.05)$ - Table 3. When CRP as

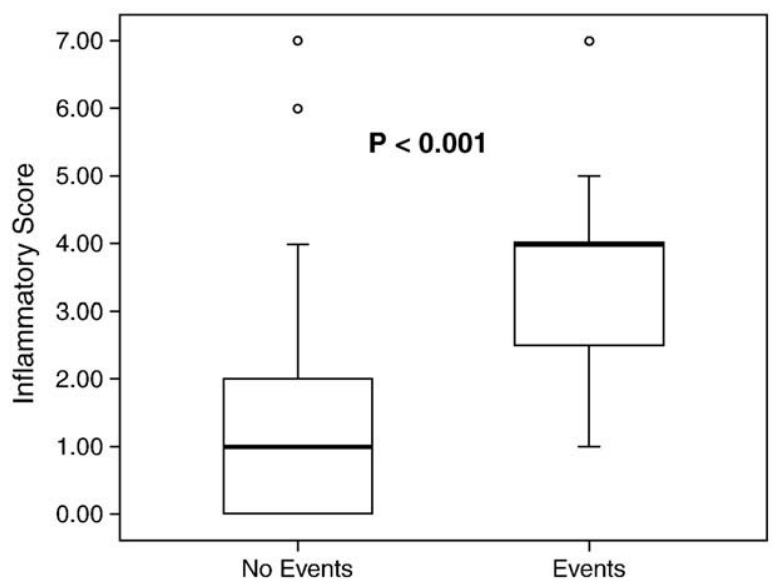

Fig. 1. Individuals who developed subsequent events have previously higher levels of the Inflammatory Score, in comparison with those free of events. 
Table 3

Logistic regression analysis adjusting the Inflammatory Score or C-reactive protein for the GRACE Score.

\begin{tabular}{llllll}
\hline $\begin{array}{l}\text { Predictive } \\
\text { variable }\end{array}$ & Beta & $\begin{array}{l}\text { Odds ratio } \\
(95 \% \mathrm{CI})\end{array}$ & $P$ value & $\begin{array}{l}P \text { value H-L test } \\
\text { (calibration) }\end{array}$ & $\begin{array}{l}C \text {-statistics } \\
(95 \% \mathrm{CI})\end{array}$ \\
\hline $\begin{array}{l}\text { GRACE Score } \\
\quad \text { (univariate) }\end{array}$ & 0.031 & $1.03(1.01-1.05)$ & 0.002 & 0.07 & $0.77(0.58-0.96)$ \\
$\begin{array}{l}\text { Model 1 } \\
\text { GRACE Score }\end{array}$ & 0.030 & $1.03(1.01-1.05)$ & 0.003 & & $0.80(0.61-0.99)$ \\
$\begin{array}{l}\text { C-reactive } \\
\text { protein }\end{array}$ & 0.017 & $1.02(1.002-1.03)$ & 0.025 & & \\
$\begin{array}{l}\text { Model 2 } \\
\text { GRACE Score }\end{array}$ & 0.024 & $1.02(1.003-1.05)$ & 0.022 & & $0.85(0.71-1.0)$ \\
$\begin{array}{l}\text { Inflammatory } \\
\quad 0.585\end{array}$ & $1.80(1.12-2.88)$ & 0.015 & & \\
$\quad$ Score & & & & & \\
\hline
\end{tabular}

Model 1 was computed by entering the GRACE Score and C-reactive protein as continuous variables.

Model 2 was computed by entering the GRACE Score and the Inflamatory Score as continuous variables.

Beta: coefficient of regression; H-L: Hosmer-Lemeshow.

a continuous variable was adjusted for the GRACE Score, this inflammatory marker also remained a significant predictor $(\mathrm{OR}=1.02 ; 95 \% \mathrm{CI}=1.002-1.03)$. Since the aim of the model was risk prediction at hospital admission, coronary angiography was not entered as a covariate.

\subsection{Additional prognostic value of inflammatory markers}

The GRACE Score had a good discriminatory ability, as expressed by a $C$-statistics of $0.77(95 \% \mathrm{CI}=0.58-0.96)$. After incorporating the Inflammatory Score into the GRACE Score, the multivariate model had a much greater $C$-statistics of $0.85(95 \% \mathrm{CI}=0.71-1.0)$, in comparison with GRACE - Table 3 and Fig. 2.

The multivariate model was translated into a GRACE-Inflammatory Score after multiplying the Inflammatory Score of each patient by the ratio of Inflammatory Score's regression coefficient/GRACE Score's regression coefficient $(0.585 / 0.024=24)$. The cut-off points of best accuracy in the ROC curve were 220 for the new score and 148 for the GRACE Score. Based on these points, the GRACE-Inflammatory Score had a better prognostic specificity in relation to GRACE ( $84 \%$ vs. $72 \%$, $P=0.01$ ), which resulted in an improved positive predicted value (43\% vs. 30\%). As opposed, sensitivity (82\%) and negative predicted

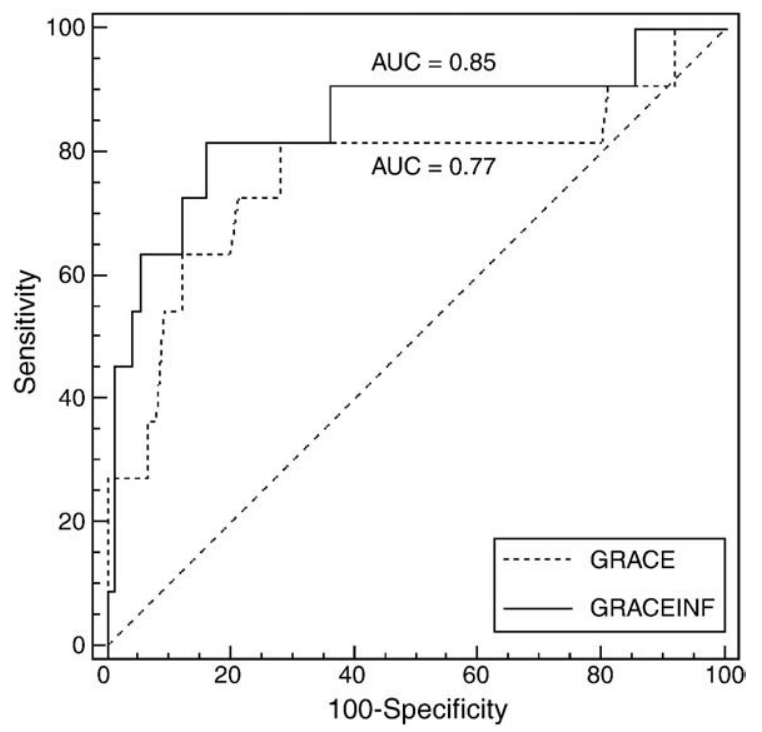

Fig. 2. AUC indicates the area under the curves ( $C$-statistics). GRACE means the receiver operator characteristic curve (ROC) of the GRACE Score. GRACEINF means GRACEInflammatory Score. A greater $C$-statistics is observed with the GRACE-Inflammatory Score, in relation to the GRACE Score. value (97\%) was exactly the same in both scores - Table 4. Accordingly, among patients without events, the GRACE-Inflammatory Score correctly reclassified 55\% of individuals, while it did not reclassify individuals with events. Net reclassification improvement obtained with GRACE-Inflammatory Score was $13 \%(P=0.007)$, indicating a significant reclassification (Table 5). In addition, Integrated Discrimination Improvement was $0.09(P=0.04)$, in comparison with GRACE Score - Table 4.

When only CRP was incorporated into the GRACE Score, the model had a C-statistics of $0.80(95 \% \mathrm{CI}=0.61-0.99)$. A GRACE-CRP Score was based on a regression coefficient ratio between CRP and GRACE of $0.57(0.017 / 0.030)$. After dichotomization by the best cut-off point (170), the GRACE-CRP Score had a trend towards improvement in specificity compared to the GRACE Score (79\% vs. $72 \%, P=0.07)$, but had the same specificity (82\%). Negative and positive predictive values of GRACE-CRP Score were $97 \%$ and $36 \%$, respectively.

\section{Discussion}

In the present study, we tested the hypothesis that a multimarker approach towards the inflammatory process improves risk prediction in patients with non-ST-elevation ACS. As a result, the inflammatory panel provided independent prognostic value in relation to the GRACE Score. In addition, after incorporation of three cytokines, three chemokines and CRP into the GRACE Score, its predictive accuracy underwent a relevant improvement, as observed by a 0.08 increase in C-statistics.

Several studies have demonstrated association of cytokines and chemokines with recurrent events in ACS, but no one quantified the additional predictive value of these markers in relation to wellvalidated clinical models. Moreover, most studies evaluated just a few cytokines together (in average two or three). Only two studies tested a pool of cytokines and chemokines. Kraaijeveld et al. evaluated 5 cytokines and 11 chemokines in 54 patients with unstable angina. CCL5 and CCL18 were associated with recurrent events in univariate analysis, but multivariate analysis was not performed [14]. Jager et al. measured several cytokines and chemokines in 44 infarcted patients, but it was a case-control study and did not evaluate risk prediction [15]. Among studies that evaluated a small number of cytokines, most performed multivariate analysis, but none tested how much improvement in C-statistics is obtained after inclusion of these markers in risk prediction [16-31]. Thus, our originality relies on the multimarker approach and on the incremental value of inflammatory markers in relation to the GRACE Score.

It should be emphasized that the inflammatory panel adds prognostic value to the strongest multivariate predictor, the GRACE Score. This score has been demonstrated to be more accurate than others, such as TIMI and PURSUIT risk scores [2,3]. In spite of that, GRACE is not a perfect prognostic model. Usually, risk stratification is designed to err on the safe side, avoiding underestimation of adverse outcomes' probability. Thus, the prediction focuses on sensitivity and often lacks specificity. Even those classified as high risk by the GRACE Score have a significant chance to remain free of a recurrent event (positive predictive value around 30\%) [9]. Therefore, whether it is

Table 4

Predictive accuracy of scores according to cut-off points.

\begin{tabular}{llllll}
\hline Models & $\begin{array}{l}\text { Cut-off } \\
\text { point }\end{array}$ & $\begin{array}{l}\text { Prognostic } \\
\text { sensitivity }\end{array}$ & $\begin{array}{l}\text { Prognostic } \\
\text { specificity }\end{array}$ & $\begin{array}{l}\text { Positive } \\
\text { predictive } \\
\text { value }\end{array}$ & $\begin{array}{l}\text { Negative } \\
\text { predictive } \\
\text { value }\end{array}$ \\
\hline GRACE & $\geq 148$ & $82 \%$ & $72 \%$ & $30 \%$ & $97 \%$ \\
GRACE-CRP & $\geq 170$ & $82 \%$ & $79 \%^{\dagger}$ & $36 \%$ & $97 \%$ \\
GRACE-Inflammatory & $\geq 220$ & $82 \%$ & $84 \%^{*}$ & $43 \%$ & $97 \%$ \\
\hline
\end{tabular}

Cut-off points of best performance according to ROC curve analysis.

* $P=0.01$ in comparison to the GRACE Score's specificity.

$+P=0.07$ in comparison to the GRACE Score's specificity. 
Table 5

Reclassification table GRACE-Inflammatory Score in relation to the GRACE Score.

\begin{tabular}{lll}
\hline & \multicolumn{2}{l}{ GRACE-Inflammatory Score } \\
\cline { 2 - 3 } GRACE Score & Low risk & High risk \\
\hline $\begin{array}{l}\text { Patients with events } \\
\text { Low risk }\end{array}$ & $2(100 \%)$ & 0 \\
High risk & 0 & $9(100 \%)$ \\
Patients without events & & \\
Low risk & $52(96 \%)$ & $2(4 \%)$ \\
High risk & $12(55 \%)$ & $10(45 \%)$ \\
\hline
\end{tabular}

Risk levels were defined by cut-off points at the ROC curve analysis.

GRACE Score $\geq 148$ defines high risk, otherwise low risk

GRACE-Inflammatory Score $\geq 220$ defines high risk, otherwise low risk.

recommended to include a new marker in the routine evaluation of ACS patients is a matter of how much improvement of specificity is obtained, because sensitivity is already good. In our study, the improvement in prognostic accuracy was mediated by an increase in specificity, which led to a better positive predictive value. Accordingly, reclassification analysis demonstrated that among individuals free of events incorrectly classified as high risk by the GRACE Score, 55\% were reclassified as low risk by the GRACE-Inflammatory Score.

The severity of ischemia during acute coronary syndromes is wellrepresented in GRACE by variables such as hemodynamic status, Killip class, myocardial necrosis markers, ST-deviation. These characteristics result from the pathological effect of aterothrombosis. However, the other piece of the puzzle, inflammation, is not contemplated by any risk score. Since inflammation delay plaque stabilization, it may contribute with recurrent events in patients with ACS [32]. This is the plausible rational of the independent association between inflammation and cardiovascular events. In fact, we previously demonstrated that CRP improves the predictive value of the TIMI-Risk score [7]. The present results indicate that the GRACE Score is also improved by CRP, but in a modest fashion. When cytokines and chemokines are added to the model, the improvement becomes relevant. This suggests that a more comprehensive evaluation of the inflammatory process may be necessary to enhance the prognostic value of a high profile score, such as GRACE.

Regarding the logistic regression analysis, we did not enter each inflammatory marker as covariates, because of intense collinearity among these variables. Instead, we opted to evaluate the utility of a cluster of biomarkers, compared with a reduced model containing only CRP as a new marker. Further studies, with greater sample sizes, should compare the predictive value of different clusters combinations, in order to establish a final model with a balance between the number of variables and the resulting predictive value. In addition to controlling for the GRACE Score, we specifically adjust the Inflammatory Score for markers of necrosis and systolic dysfunction, which are phenomena with great potential to enhance inflammation. By doing this, we demonstrated that the predictive value of inflammation takes place over and above necrosis and systolic dysfunction phenomena.

All the cytokines and chemokines studied are directly and indirectly linked to inflammatory responses. The cytokines selected are the ones that have been previously suggested as predictors of events in ACS. In addition, we originally evaluated IL-12 in order to assess the Th1 immune response. It is known that the release of IL-12 by antigen-presenting cells stimulates the production of interferon- $\gamma$ [33]. The chemokines selected are involved in migration of monocytes, neutrophils and lymphocytes, all of which presenting important role on the acute pathological processes. There are approximately 50 chemokines, but we specifically prioritize the ones that have been previously associated with coronary disease [34].

The absence of association with events of certain cytokines and chemokines merits discussion, such as IL-6, IL-10 and CCL5 (RANTES) and CXCL10. Previous literature is not definitive about the predictive value of these specific markers. Regarding IL-6, some studies have suggested its univariate association with events [19,23-25,30,31], but no independent prediction was observed after adjustment to clinical variables [26,28]. Albeit being considered an anti-inflammatory mediator, IL-10 has been shown to be elevated in a number of inflammatory disorders, such as systemic infections, sepsis and autoimmune disorders. In these cases, and probably in patients with acute cardiac diseases, an elevated IL-10 systemic level could indirectly indicate counter regulatory responses against a huge inflammatory panorama. In addition, IL-10 ratio with other inflammatory cytokines has been used as a means to reflect the equilibrium between pro-inflammatory and anti-inflammatory stimulus $[22,25,29]$. Most evidence regarding IL-10 indicates an inverse association with events $[21,22,25,27,29]$, but the largest study suggested a direct association [20]. CCL5 has been tested in only 1 study of 54 patients, which suggested a significant predictive value [14]. Regarding CXCL10, we are the first to evaluate this chemokine. Therefore, the negative results of our study for certain inflammatory markers are not in contrast with previous literature, which is controversial regarding these specific cytokines. However, considering our limited sample size, one should be aware for the possibility of type II error as an explanation of these negative findings.

While our study is a proof of concept, it is not definitive in validating the measurement of these markers in clinical practice. Before any definitive conclusion, the new score must be reevaluated in a validation set. In addition, other levels of evidence are required, such as: the new score should be able to increment GRACE's ability to predict the benefit of therapeutic strategies; randomized clinical trials should demonstrate the impact of the multimarker approach in patients' outcome; and finally, measurement of cytokines and chemokines should become more practical and cheaper, while costeffective analyses are performed. In conclusion, the present study provides preliminary evidence that a comprehensive assessment of inflammation provides additional prognostic information to the GRACE Score.

\section{Acknowledgements}

This work received financial support from Papes Fiocruz. BBA received a fellowship from the National Brazilian Research Council (CNPq). VMB and MB-N are senior fellows from CNPq.

\section{References}

[1] Yan AT, Yan RT, Huynh T, et al. Understanding physicians' risk stratification of acute coronary syndromes: insights from the Canadian ACS 2 Registry. Arch Intern Med 2009;169:372-8.

[2] de Araujo GP, Ferreira J, Aguiar C, Seabra-Gomes R. TIMI, PURSUIT, and GRACE risk scores: sustained prognostic value and interaction with revascularization in NSTEACS. Eur Heart J 2005;26:865-72.

[3] Yan AT, Yan RT, Tan M, et al. Risk scores for risk stratification in acute coronary syndromes: useful but simpler is not necessarily better. Eur Heart J 2007;28: 1072-8.

[4] Armstrong EJ, Morrow DA, Sabatine MS. Inflammatory biomarkers in acute coronary syndromes: part I: introduction and cytokines. Circulation 2006;113: e72-5.

[5] Pearson TA, Mensah GA, Alexander RW, et al. Markers of inflammation and cardiovascular disease: application to clinical and public health practice: a statement for healthcare professionals from the Centers for Disease Control and Prevention and the American Heart Association. Circulation 2003;107:499-511.

[6] O'Donoghue M, Morrow DA. The future of biomarkers in the management of patients with acute coronary syndromes. Curr Opin Cardiol 2008;23:309-14.

[7] Correia LC, Lima JC, Rocha MS, D'Oliveira JA, Pericles EJ. Does high-sensitivity C-reactive protein add prognostic value to the TIMI-Risk Score in individuals with non-ST elevation acute coronary syndromes? Clin Chim Acta 2007;375:124-8.

[8] Apple FS, Quist HE, Doyle PJ, Otto AP, Murakami MM. Plasma 99th percentile reference limits for cardiac troponin and creatine kinase MB mass for use with European Society of Cardiology/American College of Cardiology Consensus Recommendations. Clin Chem 2003;49:1331-6.

[9] Granger CB, Goldberg RJ, Dabbous O, et al. Predictors of hospital mortality in the global registry of acute coronary events. Arch Intern Med 2003;163:2345-53. 
[10] Chen R, Lowe L, Wilson JD, et al. Simultaneous quantification of six human cytokines in a single sample using microparticle-based flow cytometric technology. Clin Chem 1999;45:1693-4.

[11] Spinas GA, Keller U, Brockhaus M. Release of soluble receptors for tumor necrosis factor (TNF) in relation to circulating TNF during experimental endotoxinemia. J Clin Invest 1992;90:533-6.

[12] Rifai N, Tracy RP, Ridker PM. Clinical efficacy of an automated high-sensitivity C-reactive protein assay. Clin Chem 1999;45:2136-41.

[13] Pencina MJ, D'Agostino Sr RB, D'Agostino Jr RB, Vasan RS. Evaluating the added predictive ability of a new marker: from area under the ROC curve to reclassification and beyond. Stat Med 2008;27:157-72.

[14] Kraaijeveld AO, de Jager SCA, de Jager WJ, et al. CC chemokine ligand-5 (CCL5/ RANTES) and CC chemokine ligand-18 (CCL18/PARC) are specific markers of refractory unstable angina pectoris and are transiently raised during severe ischemic symptoms. Circulation 2007;116:1931-41.

[15] de Jager SC, Kraaijeveld AO, Grauss RW, et al. CCL3 (MIP-1 alpha) levels are elevated during acute coronary syndromes and show strong prognostic power for future ischemic events. J Mol Cell Cardiol 2008;45:446-52.

[16] de Lemos JA, Morrow DA, Sabatine MS, et al. Association between plasma levels of monocyte chemoattractant protein-1 and long-term clinical outcomes in patients with acute coronary syndromes. Circulation 2003;107:690-5.

[17] Kervinen $\mathrm{H}$, Manttari M, Kaartinen M, et al. Prognostic usefulness of plasma monocyte/macrophage and T-lymphocyte activation markers in patients with acute coronary syndromes. Am J Cardiol 2004;94:993-6.

[18] de Lemos JA, Morrow DA, Blazing MA, et al. Serial measurement of monocyte chemoattractant protein-1 after acute coronary syndromes: results from the A to Z trial. J Am Coll Cardiol 2007;50:2117-24.

[19] Jaremo P, Nilsson O. Interleukin-6 and neutrophils are associated with long-term survival after acute myocardial infarction. Eur J Intern Med 2008;19:330-3.

[20] Malarstig A, Eriksson P, Hamsten A, Lindahl B, Wallentin L, Siegbahn A. Raised interleukin-10 is an indicator of poor outcome and enhanced systemic inflammation in patients with acute coronary syndrome. Heart 2008;94:724-9.

[21] Tziakas DN, Chalikias GK, Kaski JC, et al. Inflammatory and anti-inflammatory variable clusters and risk prediction in acute coronary syndrome patients: a factor analysis approach. Atherosclerosis 2007;193:196-203.

[22] Chalikias GK, Tziakas DN, Kaski JC, et al. Interleukin-18/interleukin-10 ratio is an independent predictor of recurrent coronary events during a 1-year follow-up in patients with acute coronary syndrome. Int J Cardiol 2007;117:333-9.
[23] Kavsak PA, Ko DT, Newman AM, et al. Risk stratification for heart failure and death in an acute coronary syndrome population using inflammatory cytokines and N-terminal pro-brain natriuretic peptide. Clin Chem 2007;53:2112-8.

[24] Hartford M, Wiklund O, Mattsson HL, et al. C-reactive protein, interleukin-6, secretory phospholipase A2 group IIA and intercellular adhesion molecule- 1 in the prediction of late outcome events after acute coronary syndromes. J Intern Med 2007;262:526-36.

[25] Kilic T, Ural D, Ural E, et al. Relation between proinflammatory to antiinflammatory cytokine ratios and long-term prognosis in patients with non-ST elevation acute coronary syndrome. Heart 2006;92:1041-6.

[26] Lee KW, Lip GY, Tayebjee M, Foster W, Blann AD. Circulating endothelial cells, von Willebrand factor, interleukin-6, and prognosis in patients with acute coronary syndromes. Blood 2005;105:526-32.

[27] Heeschen C, Dimmeler S, Hamm CW, et al. Serum level of the antiinflammatory cytokine interleukin-10 is an important prognostic determinant in patients with acute coronary syndromes. Circulation 2003;107:2109-14

[28] Rallidis LS, Zolindaki MG, Manioudaki HS, Laoutaris NP, Velissaridou AH, Papasteriadis EG. Prognostic value of C-reactive protein, fibrinogen, interleukin-6, and macrophage colony stimulating factor in severe unstable angina. Clin Cardiol 2002;25:505-10.

[29] Anguera I, Miranda-Guardiola F, Bosch X, et al. Elevation of serum levels of the anti-inflammatory cytokine interleukin-10 and decreased risk of coronary events in patients with unstable angina. Am Heart J 2002;144:811-7.

[30] Koukkunen H, Penttila K, Kemppainen A, et al. C-reactive protein, fibrinogen, interleukin-6 and tumour necrosis factor-alpha in the prognostic classification of unstable angina pectoris. Ann Med 2001;33:37-47.

[31] Biasucci LM, Liuzzo G, Fantuzzi G, et al. Increasing levels of interleukin (IL)-1Ra and IL-6 during the first 2 days of hospitalization in unstable angina are associated with increased risk of in-hospital coronary events. Circulation 1999;99:2079-84.

[32] Libby P, Ridker PM, Maseri A. Inflammation and atherosclerosis. Circulation 2002;105:1135-43.

[33] Delves PJ, Roitt IM. The immune system. Second of two parts. N Engl J Med 2000;343:108-17.

[34] Aukrust P, Halvorsen B, Yndestad A, et al. Chemokines and cardiovascular risk. Arterioscler Thromb Vasc Biol 2008;28:1909-19. 